\title{
Hypergeometric foundations of Fokker-Plank like equations
}

\author{
A. Plastino ${ }^{1}$, M.C.Rocca ${ }^{1}$ \\ ${ }^{1}$ La Plata National University and Argentina's National Research Council \\ (IFLP-CCT-CONICET)-C. C. 727, 1900 La Plata - Argentina
}

June 28, 2021

\begin{abstract}
We show that the Fokker Planck equation can be derived from a Hypergeometric differential equation. The same applies to a non linear generalization of such equation.
\end{abstract}

Keywords: Non linear Fokker-Plank equations, separation of variables, hypergeometric function. 


\section{Introduction}

In this paper we uncover the fact that the celebrated Fokker-Planck (FP) equation [1]

$$
\frac{\partial F}{\partial t}=-\frac{\partial}{\partial x}[K(x) F]+\frac{Q}{2} \frac{\partial^{2} F}{\partial x^{2}},
$$

can be derived from an hypergeometric differential equation. In this equation, $\mathrm{F}$ is the distribution function, $\mathrm{K}(\mathrm{x})$ the drift coefficient and $\mathrm{Q}$ the diffusion coefficient (a positive quantity) [1]. The second term on the r.h.s, describes the effects of the fluctuating forces (diffusion term). Without it, (1.1) would describe deterministic motion (over-damped motion of a particle under the force $K(x)$ ). For the time being, we restrict ourselves to the case $\mathrm{K}=$ constant. A similar hypergeometric derivation applies to a non linear generalization of equation (1.1), in the spirit if the one advanced 20 years ago by Plastino and Plastino [2], that has arisen interest till today [4, 5, 6, 7]. This papers continues a line of research initiated by uncovering hypergeometric connotations of quantum equations [3].

\section{Hypergeometric derivation of the Fokker- Plank equation}

The ordinary hypergeometric function $\mathrm{F}_{1}^{2}(\mathrm{a}, \mathrm{b} ; \mathrm{c} ; z)$ is a special function represented by the hypergeometric series, that includes many other special functions as specific or limiting cases. It is a solution of a second-order linear ordinary differential equation (ODE). Many second-order linear ODEs can be transformed into this equation. Generalized hypergeometric functions include the confluent hypergeometric function as a special case, which in turn have many particular special functions as special instances, such as elementary functions, Bessel functions, and the classical orthogonal polynomials. In particular, the confluent hypergeometric function reads [3]

$$
\phi(a, b, z)=\sum_{n=0}^{\infty} \frac{a_{n}}{b_{n}} \frac{z^{n}}{n !} ; a, b \in \mathcal{R}
$$

with $a_{n}, b_{n}$ the Pochhamer symbols 


$$
a_{0}=1, a_{n}=a(a+1)(a+2) \ldots(a+n-1) \text {; same for } b .
$$

The confluent hypergeometric equation satisfies the differential equation [3]:

$$
z \phi^{\prime \prime}(a, b, z)+(b-z) \phi^{\prime}(a, b, z)-a \phi(a, b, z)=0
$$

that, for $\mathrm{a}=\mathrm{b}$ adopts the appearance

$$
z \phi^{\prime \prime}(a, a, z)+(a-z) \phi^{\prime}(a, a, z)-a \phi(a, a, z)=0 .
$$

Accordingly,

$$
\phi(a, a, z)=e^{z}
$$

Let us consider now the function $\phi$ and the variable $\lambda$

$$
\phi\left[a, a,-\left(\lambda t+\frac{x}{\lambda}\right)\right]=e^{-\left(\lambda t+\frac{x}{\lambda}\right)}
$$

where we express $\lambda$ in terms of an equation involving two quantities $K$ and Q of Eq. (1.1)

$$
\lambda^{3}+\mathrm{K} \lambda+\frac{\mathrm{Q}}{2}=0
$$

and we define $z$ as

$$
z=-\lambda t-\frac{x}{\lambda}
$$

Given that $\phi$ is such that

$$
\phi^{\prime \prime}=\lambda^{2} \frac{\partial^{2} \phi}{\partial x^{2}} \quad ; \quad \phi^{\prime}=-\frac{1}{\lambda} \frac{\partial \phi}{\partial t} \equiv \phi
$$

Eq. (2.3) can be recast as

$$
z \lambda^{2} \frac{\partial^{2} \phi}{\partial x^{2}}+a \phi^{\prime}+\frac{z}{\lambda} \frac{\partial \phi}{\partial t}-a \phi=0 .
$$

Since $\phi^{\prime}=\phi,(2.9)$ gets simplified to

$$
\lambda^{3} \frac{\partial^{2} \phi}{\partial x^{2}}+\frac{\partial \phi}{\partial t}=0 .
$$

According to (2.6), Eq. (2.10) becomes

$$
-\left(K \lambda+\frac{Q}{2}\right) \frac{\partial^{2} \phi}{\partial x^{2}}+\frac{\partial \phi}{\partial t}=0 .
$$


In addition, since $\phi$ verifies

$$
\lambda \frac{\partial^{2} \phi}{\partial x^{2}}=-\frac{\partial \phi}{\partial x}
$$

we are led to the following expression for (2.11)

$$
K \frac{\partial \phi}{\partial x}-\frac{Q}{2} \frac{\partial^{2} \phi}{\partial x^{2}}+\frac{\partial \phi}{\partial t}=0
$$

which is tantamount to

$$
\frac{\partial \phi}{\partial t}+\frac{\partial(K \phi)}{\partial x}-\frac{Q}{2} \frac{\partial^{2} \phi}{\partial x^{2}}=0
$$

i.e., Fokker-Plank's equation para K independent of $x$. Of course, when K does depend upon $x$ one just postulates (2.14). Note that, by definition, (2.5) is a solution of (2.14).

\section{Separation of variables in the Ornstein-Uhlenbeck process $\mathrm{K}=x$}

The OrnsteinUhlenbeck process is a stochastic process that, loosely, describes the velocity of a massive Brownian particle under the influence of friction. It is stationary, Gaussian, and Markovian, being the only nontrivial evolution

that satisfies these three conditions, up to allowing for linear transformations of the space and time variables. We believe that this well known process of linear drift [1] is worth revisiting for didactic purposes. We start with

$$
\begin{gathered}
\frac{\partial F}{\partial t}+\frac{\partial(K F)}{\partial x}-\frac{Q}{2} \frac{\partial^{2} F}{\partial x^{2}}=0, \\
F(x, t)=G(t) H(x),
\end{gathered}
$$

which leads to

$$
\frac{1}{\mathrm{G}} \frac{\partial \mathrm{G}}{\partial \mathrm{t}}=\frac{1}{\mathrm{H}}\left[\frac{\mathrm{Q}}{2} \frac{\partial^{2} \mathrm{H}}{\partial x^{2}}-\frac{\partial(\mathrm{KH})}{\partial x}\right]=-\lambda,
$$

with $\lambda>0$. From here we are immediately led to

$$
\frac{\partial G}{\partial t}+\lambda G=0
$$




$$
\frac{\mathrm{Q}}{2} \frac{\mathrm{d}^{2} \mathrm{H}}{\mathrm{d} x^{2}}-\frac{\mathrm{d}(\mathrm{KH})}{\mathrm{d} x}+\lambda \mathrm{H}=0 .
$$

For the linear instance $K=-\chi$ we first obtain for $G$

$$
\mathrm{G}(\mathrm{t})=\mathrm{e}^{-\lambda \mathrm{t}} .
$$

Applying the Fourier transform to (3.5) we find

$$
\frac{\mathrm{Q}}{2} \alpha^{2} \hat{\mathrm{H}}+\alpha \frac{\mathrm{d} \hat{\mathrm{H}}}{\mathrm{d} \alpha}-\lambda \hat{\mathrm{H}}=0,
$$

where $\hat{\mathrm{H}}$ is the Fourier transform of $\mathrm{H}$ of variable $\alpha$. One solves (3.7) and get

$$
\hat{H}(\alpha)=|\alpha|^{\lambda} e^{-\frac{Q \alpha^{2}}{4}},
$$

and from (3.8) we encounter for $\mathrm{H}$

$$
H(x)=\frac{1}{2 \pi} \int_{-\infty}^{\infty}|\alpha|^{\lambda} e^{-\frac{Q \alpha^{2}}{4}} e^{-i \alpha x} \mathrm{~d} \alpha .
$$

Thus we have for $\mathrm{F}$ the general expression

$$
F(x, t)=\frac{1}{2 \pi} \int_{0}^{\infty} \int_{-\infty}^{\infty} \lambda a(\lambda) e^{-\lambda t}|\alpha|^{\lambda} e^{-\frac{Q \alpha^{2}}{4}} e^{-i \alpha x} d \alpha d \lambda,
$$

where $a(\lambda)$ must verify

$$
\int_{0}^{\infty} a(\lambda) d \lambda=1 .
$$

Eq. (3.10) may have been obtained before, but we were unable to find such derivation in the vast FP-literature available to us.

\section{Non-Linear Fokker-Plank Equation [8]}

Anomalous diffusion is exhibited in a variety of physical systems and is therefore the subject of much interest. It can be observed, for example, in general systems such as plasma flow, porous media, and surface growth, as well 
as in more specific situations such as cytltrimethylammonium bromide miscelles dissolved in salted water and NMR relaxometry of liquids in porous glasses [8. The main characteristic of anomalous diffusion is the fact that the mean squared displacement is not proportional to time $t$ but rather to some power of it. If the scaling is faster than $t$, then the pertinent system is superdiffusive while, if it is slower than $t$, it is subdiffusive. A nonlinear Fokker-Planck diffusion equation has been proposed for those systems with correlated anomalous diffusion, beginning with [2] and followed afterward by, for instance, [9, 10, 11]. For an excellent overview, see [8].

For the ordinary hypergeometric function $\mathrm{F}_{1}^{2}(\mathrm{a}, \mathrm{b} ; \mathrm{c} ; z)$ we have [12], using now three Pochhamer symbols,

$$
\mathrm{F}_{1}^{2}(\mathrm{a}, \mathrm{b} ; \mathrm{c} ; z) \equiv \mathrm{F}(\mathrm{a}, \mathrm{b} ; \mathrm{c} ; z)=\sum_{\mathrm{n}=0}^{\infty} \frac{\mathrm{a}_{(\mathfrak{n})} \mathrm{b}_{(\mathfrak{n})}}{\mathrm{c}_{(\mathrm{n})}} \frac{z^{\mathfrak{n}}}{\mathrm{n} !} ;(|z|<1),
$$

where the series terminates if either $\mathrm{a}$ or $\mathrm{b}$ is a non-zero integer. A particularly important special case is

$$
\mathrm{F}(-\mathrm{m}, \mathrm{b}, \mathrm{b},-z)=(1+z)^{\mathrm{m}} .
$$

Eq. (4.1) verifies 12

$z(1-z) \mathrm{F}^{\prime \prime}(\alpha, \beta ; \gamma ; z)+[\gamma-(\alpha+\beta+1) z] \mathrm{F}^{\prime}(\alpha, \beta ; \gamma ; z)-\alpha \beta \mathrm{F}(\alpha, \beta ; \gamma ; z)=0$.

If $\beta=\gamma$, then $\mathrm{F}$ satisfies 13

$$
\mathrm{F}(-\alpha, \gamma ; \gamma ;-z)=(1+z)^{\alpha} .
$$

Focus attention now upon the function

$$
f(x, t)=\left[1+(q-1)\left(\lambda t+\frac{x}{\lambda}\right)\right]^{\frac{1}{1-q}},
$$

where $\lambda$ obeys (for $\mathrm{K}$ and $\mathrm{Q}$ both constants)

$$
\lambda^{3}+\mathrm{K} \lambda+\frac{\mathrm{Q}}{2}=0 .
$$

Recourse to (4.4) allows one to write

$$
F\left[\frac{1}{q-1}, \gamma ; \gamma ;(1-q)\left(\lambda t+\frac{\chi}{\lambda}\right)\right]=\left[1+(q-1)\left(\lambda t+\frac{\chi}{\lambda}\right)\right]^{\frac{1}{1-q}},
$$


and then

$$
z=(1-q)\left(\lambda t+\frac{x}{\lambda}\right)
$$

For $\beta=\gamma, F[$ Cf. (4.3)] $]$ adopts the appearance

$z(1-z) \mathrm{F}^{\prime \prime}(\alpha, \gamma ; \gamma ; z)+[\gamma-(\alpha+\gamma+1) z] \mathrm{F}^{\prime}(\alpha, \gamma ; \gamma ; z)-\alpha \beta \mathrm{F}(\alpha, \gamma ; \gamma ; z)=0$

Since $F$ verifies

$$
F^{\prime \prime}=\frac{\lambda^{2}}{(1-q)^{2}} \frac{\partial^{2} F}{\partial x^{2}} ; F^{\prime}=\frac{1}{\lambda} \frac{\partial F}{\partial t}
$$

then (4.9) becomes

$$
z(1-z) \frac{\lambda^{2}}{(1-q)^{2}} \frac{\partial^{2} F}{\partial x^{2}}+\frac{q z}{\lambda(1-q)^{2}} \frac{\partial F}{\partial t}+\gamma(1-z) F^{\prime}-\frac{\gamma}{q-1} F=0,
$$

and, adequately simplifying,

$$
(1-z) \lambda^{3} \frac{\partial^{2} \mathrm{~F}}{\partial x^{2}}+q \frac{\partial F}{\partial t}+\frac{\gamma}{z}(1-q)^{2}\left[(1-z) F^{\prime}-\frac{1}{q-1} F\right]=0 .
$$

Again, since F fulfills

$$
(1-z) \mathrm{F}^{\prime}-\frac{1}{\mathrm{q}-1} \mathrm{~F}=0
$$

Eq. (4.12) becomes

$$
(1-z) \lambda^{3} \frac{\partial^{2} F}{\partial x^{2}}+q \frac{\partial F}{\partial t}=0
$$

or, equivalently,

$$
\lambda^{3} F^{(1-q)} \frac{\partial^{2} F}{\partial x^{2}}+q \frac{\partial F}{\partial t}=0
$$

since $F^{(1-q)}(z)=1-z$. Thus, we are in a position to cast (4.15) as

$$
\lambda^{3} \frac{\partial^{2} F}{\partial x^{2}}+\frac{\partial F q}{\partial t}=0
$$

Utilizing (4.6) we can recast things as

$$
-\left(\lambda K+\frac{Q}{2}\right) \frac{\partial^{2} F}{\partial x^{2}}+\frac{\partial F^{q}}{\partial t}=0
$$


Remembering that $\mathrm{F}$ obeys

$$
\lambda K \frac{\partial^{2} F}{\partial x^{2}}=-K \frac{\partial F^{q}}{\partial x}=-\frac{\partial\left(K F^{q}\right)}{\partial x}
$$

we obtain from (4.17)

$$
\frac{\partial F^{q}}{\partial t}+\frac{\partial\left(K F^{q}\right)}{\partial x}-\frac{Q}{2} \frac{\partial^{2} F}{\partial x^{2}}=0
$$

a nonlinear Fokker-Planck equation. We postulate its validity for $\mathrm{K}=\mathrm{K}(\mathrm{x})$ as well. If we set

- $\mathrm{g}=\mathrm{F}^{\mathrm{q}}$

- $2-\mathrm{q}^{*}=1 / \mathrm{q}$,

we immediately ascertain that Eq. (4.19), expressed in terms of $\mathrm{g}$ and $\mathrm{q}^{*}$, coincides with the nonlinear FP postulated by Plastino and Plastino in [2]

$$
\frac{\partial g}{\partial t}+\frac{\partial(K g)}{\partial x}-\frac{Q}{2} \frac{\partial^{2} g^{2-q^{*}}}{\partial x^{2}}=0
$$

For the stationary case ( $F$ independent of $t$ ) we have for Eq. (4.19)

$$
\frac{\partial\left(K(x) F^{q}\right)}{\partial x}-\frac{Q}{2} \frac{\partial^{2} F}{\partial x^{2}}=0,
$$

whose solution is

$$
F(x)=\left[1+\frac{2(q-1)}{Q} V(x)\right]^{\frac{1}{1-q}},
$$

where $\frac{d V(x)}{d x}=-K(x)$.

\section{Conclusions}

We have shown that the Fokker-Planck equation and its nonlinear generalization by Plastino and Plastino 2] are contained within the structure of hypergeometric linear differential equations, for constant drift $\mathrm{K}$. The FPextensions to general drifts $\mathrm{K}(x)$ have to be postulated like in the ordinary cases.

We have displayed a general solution for the Orstein-Uhlenbeck equation of constant drift that possibly might be new, although we cannot ascertain it. We also give an exact solution of the nonlinear FP equation when F does not depend upon the time. 


\section{References}

[1] H. Risken, The Fokker-Planck equation (Springer, Berlin, 1989).

[2] A. R. Plastino, A. Plastino, Physica A 222 (1995) 347.

[3] A. Plastino and M. C. Rocca:"Hypergeometric Connotations of Quantum Equations". ArXiV: 1505.06365; A.Plastino and M. C. Rocca: Physics Letters A, 379 (2015) 2690.

[4] M. N. Najafi, Phys. Rev. E 92, 022113 (2015)

[5] Z. G. Arenas, D. G. Barci, C. Tsallis Phys. Rev. E 90, 032118 (2014).

[6] G. A. Casas, F. D. Nobre, E. M. F. Curado Phys. Rev. E 86, 061136 (2012).

[7] M. S. Ribeiro, C. Tsallis, F. D. Nobre, Phys. Rev. E 88, 052107 (2013);

M. S. Ribeiro, F. D. Nobre, C. Tsallis, ibid. 89, 052135 (2014).

[8] T. D. Frank, The nonlinear Fokker-Planck equation (Springer, Berlin, 2005).

[9] C. Tsallis, D. J. Bukman, Phys. Rev. E 54 (1996) R2197 1996; A. Compte, D. Jou, J. Phys. A 29 (1996) 4321; A. Compte, D. Jou, Y. Katayama, J. Phys. A 30 (19997) 1023.

[10] L. Borland, Phys. Rev. E 57 (1998) 6634.

[11] V. Schwammle, E.M.F. Curado, F.D. Nobre, Eur. Phys. J. B 58 (2007) 159.

[12] I. S. Gradshteyn and I. M. Ryzhik: "Table of Integtals, Series and Products", 9.151, p.1045. Academic Press (1965).

[13] M. Abramowitz and I. Stegun: "Handbook of Mathematical Functions", 15.1.8, p.556. National Bureau of Standards Applied Mathematics Series 55 (1964). 